FOLIA

Amazónico

Revista del Instituto de Investigaciones

de la Amazonía Peruana

\title{
REPIQUETES Y RIESGO EN EL CULTIVO DE ARROZ EN LA LLANURA INUNDABLE DEL RÍO AMAZONAS CERCA DE IQUITOS, PERÚ
}

\author{
Geneva LIST ${ }^{1}$ y Oliver T. COOMES ${ }^{1}$ \\ ${ }^{1}$ Departamento de Geografía, Burnside Hall, Room 705, McGill University, 805 Sherbrooke Street \\ West, Montreal, Quebec H3A 0B9. Correo electrónico: geneva.list@mail.mcgill.ca
}

\section{RESUMEN}

Las llanuras inundables del río Amazonas poseen un gran potencial para la agricultura, pero no están exentas de riesgos para los agricultores que cultivan en esas tierras fértiles muy propensas a inundaciones. Estasinundaciones repentinas, que ocurren a medida que el nivel del río disminuye entre mayo y noviembre, son conocidas como «repiquetes» y representan una amenaza seria para los cultivos en llanuras inundables, especialmente para el arroz comercial. En este artículo se analiza el registro diario de los niveles de agua del río Amazonas en Iquitos de los últimos 45 años (entre 1968 y 2012), para determinar la frecuencia y magnitud de los repiquetes y su impacto en la temporada de crecimiento del cultivo. Las entrevistas y los estudios de campo realizados en cuatro comunidades ribereñas, cerca del archipiélago Muyuy, revelan el impacto de los repiquetes y otros riesgos en la producción de arroz, así como la disposición de los agricultores al pago por los instrumentos apropiados para disminuir el riesgo de las inundaciones. Nuestros hallazgos apuntan a un seguro basado en índices climáticos, como una nueva estrategia para reducir el riesgo y promover el desarrollo agrícola en las llanuras inundables del Amazonas.

PALABRAS CLAVE: Agricultura de recesión por inundaciones, arroz, análisis de riesgos, inundaciones, pérdida de cultivos, estrategias de adaptación, Amazonía. 


\title{
REPIQUETES AND RISK IN THE CULTIVATION OF RICE IN THE FLOODPLAIN OF THE AMAZON RIVER NEAR IQUITOS, PERU
}

\begin{abstract}
The floodplain of the Amazon river offers considerable potential for agriculture but is a risk-filled environment for farmers working the fertile but flood-prone land. Flood reversals that occur as river levels fall and rise between May and November - known as repiquetes - pose a serious threat to crop cultivated on the floodplains, particularly commercial rice. In this paper we analyze the 45 -year record of daily water levels at Iquitos to determine the frequency and magnitude of repiquetes and their impact on the crop growing season. Interviews and land surveys conducted in four riverside communities near the Isla Muyuy archipelago indicate the impact of repiquetes and other hazards on rice production, and assessed farmers' willingness to pay for flood risk adaptation tools. Our findings point to index-based insurance as promising strategy to buffer risk and promote agricultural development of the Amazon floodplain.

KEY WORDS: Flood recession agriculture, rice, risk analysis, flooding, crop loss, adaptation strategies, Amazonia.
\end{abstract}




\section{INTRODUCCIÓN}

Los agricultores que cultivan en llanuras inundables de los principales ríos tropicales han empleado durante mucho tiempo estrategias de cultivo asociadas con el ciclo anual de las inundaciones para el mejoramiento de su productividad. A lo largo del río Amazonas en Perú, los agricultores, que viven en las llanuras inundables y las comunidades de tierras altas, siembran diferentes cultivos para subsistir y obtener ingresos económicos, siendo el arroz de tierras inundables el cultivo comercial más importante (Chibnik, 1994). Aunque la agricultura en suelos inundables tiene mayores rendimientos que en tierra firme, los agricultores enfrentan el riesgo de pérdida de sus sembríos debido a las inundaciones. Al reducirse la temporada de crecimiento de los cultivos, se ponen en peligro los medios de subsistencia de los agricultores de los llanos inundables, lo que es un impedimento importante para el desarrollo del potencial agrícola de las tierras bajas de la Amazonía (Petrick, 1978; Chibnik, 1994; Smith et al., 1995; Labarta et al., 2007).

Además del pulso de inundación anual, las reversiones intempestivas en la dirección de los cambios de estadío del río conocidas como "repiquetes", que ocurren a lo largo del río Amazonas, tienen efectos importantes en los ecosistemas ribereños, la vida acuática y la agricultura de recesión en las tierrasbajas (Coomes et al., 2016). Las reversiones a escala semanal en la tendencia estacional del recambio ocurren regularmente durante las etapas anuales, tanto cuando el río está mermando como cuando está subiendo. En el largo trayecto del río Amazonas, el amplio período de tiempo transcurrido entre sus principales tributarios zonal y meridional, favorece la asincronía de los afluentes y hace que los repiquetes sean comunes. En cuanto las aguas de inundación retroceden y los agricultores inician la siembra en suelos aluviales recién expuestos, los repiquetes exponen las semillas sin germinar a ser consumidas por los peces o para que los suelos anegados las entierren con sedimentos o ahoguen las plantas jóvenes (Ríos Arévalo, 2005). Cuando las aguas comienzan a elevarse después de alcanzar su punto más bajo, las reversiones del curso del río ahogan los cultivos inmaduros en las llanuras aluviales. Es importante prestar atención $-y$ con frecuencia se suele pasar por alto- el hecho de que las inundaciones acortan el período de exposición del suelo libre de agua, $y$, por lo tanto, la temporada de crecimiento de las plantas. Los agricultores siembran sus cultivos en zonas elevadas, aquellos de maduración más lenta en suelos de mayor elevación (p.e., en restingas altas: plátano, cultivos de árboles perennes) y los de maduración más rápida en suelos de menor elevación (p.e., barreales: arroz; playas: chiclayo) (Denevan, 1984).

La breve temporada de crecimiento de cultivo a lo largo del río Amazonas en el norte de la Amazonía peruana - por lo general de junio a octubre - y la amenaza de inundaciones adelantadas, son limitaciones importantes para la producción agrícola en la llanura inundable en los alrededores de Iquitos (Chibnik, 1994). La creciente del río Amazonas eleva su nivel durante los meses de marzo a mayo y alcanza su mínimo en septiembre. De acuerdo con la Estación de Medición de ENAPU del Servicio de Hidrografía y Navegación de la Amazonía (SEHINAV/Marina de Guerra del Perú), los niveles del río en Iquitos varían fluctuando aproximadamente nueve metros por año (108-117 msnm) y el tiempo de subida y bajada del río varían considerablemente año tras año debido a las condiciones climáticas globales y regionales. Los modelos simulados de cambio climático predicen un aumento del área inundada de la Amazonía occidental con una duración de la inundación de 2 a 3 meses, y con un incremento en los años lluviosos extremos que 
persisten mucho más en el noroeste (Langerwisch et al., 2013). Los análisis empíricos de los datos del nivel de agua diario, reportados por el SENAMHI en Tamshiyacu, indican desde 1985 una tendencia a la disminución de los niveles de agua en la vaciante, un incremento en la duración de la temporada de vaciante y un incremento del nivel de agua en la creciente (Ronchail et al., 2018). Claramente se necesitarán nuevas estrategias y herramientas de gestión de riesgos para permitir que los agricultores se adapten a los futuros cambios hidrológicos del río Amazonas, como también para fomentar prácticas de cultivo con mejor información y una mayor inversión en la agricultura de la llanura inundable.

\section{MATERIALES Y MÉTODOS}

Los datos se recopilaron en cuatro comunidades agricultoras: Cantagallo, Mazanillo, Dos de Mayo y Augusto Freyre, ubicadas de 5 a 20 kilómetros al sur de Iquitos, a lo largo de la ribera izquierda del río Amazonas y cerca al archipiélago de Muyuy. Esta ramificación del río Amazonas - que forma casi una decena de islas y pequeñas lagunas en su interior - tiene altas tasas de sedimentación y erosión (Mendoza et al., 2016). Para evaluar la topografía local y la extensión del terreno de las áreas potenciales para el cultivo de arroz examinamos cuatro secciones transversales de barreales, utilizando como punto de referencia de la elevación el nivel del agua registrado a $20 \mathrm{~km}$ aguas arriba del río, por el SEHINAV en Iquitos.

En cada comunidad condujimos encuestas en hogares, entre junio y septiembre de 2014, entrevistando a todas las cabezas de familia disponibles $(\mathrm{n}=83 ; 90 \%$ del total $)$ queconsintieron participar del proyecto brindando información voluntaria, como parte de los requisitos del Consejo de Ética de Investigación de la Universidad de McGill (Archivo 475-0514). El cuestionario semiestructurado solicitó información sobre la demografía de los hogares, ingresos (subsistencia y dinero en efectivo), propiedad de la tierra, prácticas agrícolas y percepciones sobre el riesgo de producción. En la encuesta se utilizó i) un método de la estimación hipotético para conseguir la disposición de los agricultores a pagar (Willingness to Pay: Disposición a Pagar) por el crédito; ii) variedades de arroz tolerantes a las inundaciones; iii) servicios de información fluvial; y iv) seguro contra inundaciones a través del enfoque de preferencia declarada de valoración contingente (ver List et al., 2019 para más detalles). La explicación hipotética para el seguro contra inundaciones agrícolas asumió un producto de seguro basado en índices climáticos, empleando el nivel de las aguas del río Amazonas como tal indicador. Se llevaron a cabo discusiones de grupos focales (focus groups) con agricultores de arroz en cada comunidad, utilizando técnicas rápidas de evaluación rural que incluían calendarios de inundaciones y cultivos y un ejercicio de clasificación participativo del riesgo de peligro.

\section{RESULTADOS}

\section{PRODUCCIÓN DE ARROZ}

El cultivo de arroz en los barreales a lo largo del río Amazonas es el cultivo agrícola más importante entre los hogares de la muestra delárea de estudio, que contribuye con alrededor de \$US 108,000 (2.8 NS/\$US, julio de 2014) a la economía familiar en 2013, lo que equivale al $44 \%$ del total ingresos agrícolas. El 45\% de las familias de la muestra (de un total de 83 familias muestreadas) cultivaron arroz en la temporada 2013 en un área total de 76 ha en el canal activo del río (Tabla 1). Las tasas de aplicación de semillas y las áreas sembradas variaron significativamente según la comunidad, con un promedio de $71 \mathrm{~kg} / \mathrm{ha}$ y $2.1 \mathrm{ha} /$ hogar. 
Los productores de arroz sembraron conforme a la fecha y la exposición de los barreales. La fecha promedio de la primera siembra fue entre el 11 y 25 de junio, para las cuatro comunidades, no obstante algunas familias continuaron sembrando hasta inicios de julio. Aunque la mayoría de las familias siembran arroz solo una vez, algunos siembran hasta cinco veces durante la misma temporada, plantando en elevaciones cada vez más bajas a medida que el río se retira. Los arroceros sembraron tantas variedades locales, como variedades de arroz certificado

Tabla 1: Producción de arroz por comunidad de estudio, río Amazonas, Perú, 2013.

\begin{tabular}{lcccc}
\hline & Cantagallo & Manzanillo & $\begin{array}{c}\text { Dos de } \\
\text { Mayo }\end{array}$ & $\begin{array}{c}\text { Augusto } \\
\text { Freyre }\end{array}$ \\
\hline Superficie total sembrada (ha) & 9 & 6 & 52 & 9 \\
Fecha de la primera siembra (promedio) & 11-Jun & 11-Jun & 25 -Jun & 18-Jun \\
Área de semilla sembrada/familia (ha) (promedio) & 2.2 & 0.68 & 3.5 & 1.1 \\
Cantidad de semilla sembrada kg/ha (promedio) & 21 & 109 & 41 & 110 \\
Fecha de cosecha (promedio) & $24-$ Sep & $8-$-ct & $24-$ Sep & 1 -Oct \\
Rendimiento (kg/ha) (promedio) & 1031 & 2819 & 3407 & 3122 \\
Cosecha/familia (kg) (promedio) & 640 & 1739 & 6553 & 839 \\
Ingresos por cosecha de arroz por familia (\$US) & $\$ 250$ & $\$ 625$ & $\$ 2,679$ & $\$ 491$ \\
(promedio) & & & & 64 \\
\% de familias que cultivan arroz & 19 & 50 & 50 & 14 \\
Numero de familias encuestadas & 21 & 18 & 30 & \\
\hline
\end{tabular}

Tabla 2: Variedades de arroz plantadas por agricultores en las comunidades de estudio, 2013.

\begin{tabular}{lcccc}
\hline & $\begin{array}{c}\text { Número de Familias } \\
\text { Sembrando Variedad } \\
\text { de Arroz }\end{array}$ & $\begin{array}{c}\text { Semillas } \\
\text { certificadas }\end{array}$ & $\begin{array}{c}\text { Días de } \\
\text { Maduración }\end{array}$ & $\begin{array}{c}\text { Rendimiento } \\
\text { promedio } \\
\text { (kg/ha) }\end{array}$ \\
\hline Aguja & 11 & No & 75 & 3685 \\
Capirona & 11 & Sí & $120-150$ & 2641 \\
Esperanza & 5 & Sí & $140-150$ & 4882 \\
Milagrito & 5 & No & 90 & 3375 \\
Tres Mesinos & 3 & No & 90 & 1458 \\
Dos Meses y Medio & 3 & No & 75 & 467 \\
Eco Arroz & 1 & Sí & 105 & 500 \\
Arroz Inti & 1 & Sí & $150-165$ & 250 \\
\hline
\end{tabular}

Notas: Los rendimientos promedio se basan en datos de encuestas de hogares y no incluyen casos de pérdida total de cultivos. Los valores del arroz Inti se registran por 2012. 
promocionadas por el gobierno, las instituciones de investigación o el sector privado (Tabla 2). Las variedades locales suelen ser de corta duración para la maduración (75-90 días), mientras que las variedades certificadas varían de mediana a larga duración (105-165 días).

La cosecha de arroz se efectuó en las cuatro comunidades a fines de septiembre y principios de octubre (media: 1 de octubre de 2013) (Tabla 1). Las cosechas variaron ampliamente entre las familias y comunidades, de 640 a 6,553 kg/familia/ comunidad con una media de $3,353 \mathrm{~kg} /$ familia. El rendimiento promedio del arroz fue de 2.93 toneladas /ha, muy por debajo de los rendimientos de 5 a 11 toneladas/ha que se esperan para las variedades certificadas (Tabla 2), pero similar al rendimiento en otras partes de la región de Loreto (es decir, 3.0 toneladas/ha, ver Humala Tasso et al., 2013). Las ganancias promedio de las familias productoras de arroz fueron de US\$ 1,339 o su equivalente al $57 \%$ del ingreso total, a un valor de mercado de US\$ 0.86 (NS/2.5) por kilogramo, en Iquitos. Las ganancias brutas por hectárea para los productores de arroz promediaron US\$2,315/ha.

\section{REPIQUETES}

De acuerdo con los datos diarios de nivel de agua proporcionados por SEHINVA, los niveles máximos y mínimos de los ríos registrados durante el registro de 45 años, entre 1968 y 2012, fueron de $118.97 \mathrm{~m}$ y 105.38 msnm respectivamente. Los

105.0

105.0 barreales comienzan a exponerse con el retroceso de las aguas del río, y la siembra de arroz se inicia después que se seca el limo, luego de una semana de la exposición del barreal. Nuestras entrevistas revelan que la siembra de arroz en el área de Isla Muyuy ocurre principalmente entre la última semana de mayo y la primera semana de Julio, en superficies de barreal que se extienden típicamente entre 116 y 112 msnm. Aunque la siembra de arroz es poco común por debajo de los 112 m (Ríos Arévalo, 2005; List, 2016), los

Figura 1. Nivel diario del río Amazonas en la estación de medición ENAPU, lquitos.


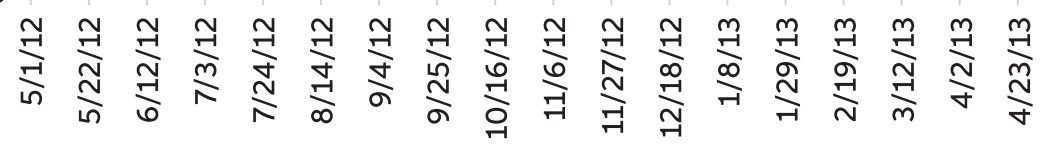


episodios de retroceso del río continúan cayendo desde finales de junio a septiembre.

Los repiquetes son comunes, pero ocurren aleatoriamente en el alcance del estudio, impulsados por los eventos de inundación que se producen en los ríos Ucayali y Marañón. Los repiquetes son visibles en hidrogramas multianuales y anuales (Figura 1A y 1B). Dentro de la síntesis récord de cinco años presentada en la Figura 1A, son evidentes los cambios de frecuencia y amplitud variables. Se pueden observar cinco eventos de repiquetes en el hidrograma para 2012-13 (Figura 1B). La Tabla 3 confirma la alta probabilidad (42-76\%) de tener al menos un repiquete durante cada uno de los principales meses de siembra de arroz (mayo, junio y julio). En junio y julio, los principales meses de siembra, cuando ocurre un repiquete, en promedio dura de 5 a 6 días (algunos hasta 20 días) antes de que los episodios de retroceso reanuden su caída. El nivel de subida promedio del repiquete durante los meses de siembra es de aproximadamente medio metro (Tabla 3). Como se ve, ningún mes de siembra está predeciblemente libre de repiquetes (es decir que tenga probabilidad 0); siempre se ve afectado por un repiquete (lo que implica descartar la siembra de arroz ese mes). De hecho, la probabilidad de que ocurra un repiquete durante los meses de septiembre, octubre y noviembre, cuando los niveles de los ríos aumentan es del $100 \%$, con el efecto de truncar la temporada de crecimiento. La probabilidad de que las aguas del río vuelvan a sumergirse en el cultivo varía notablemente según la elevación del terreno (ver Figura 2). Mientras que el riesgo de inundación es relativamente bajo en elevaciones por encima de $114 \mathrm{~m}$, el riesgo aumenta bruscamente en elevaciones menores,

Figura 2: Probabilidad de resubmergencia por nivel del río Amazonas.

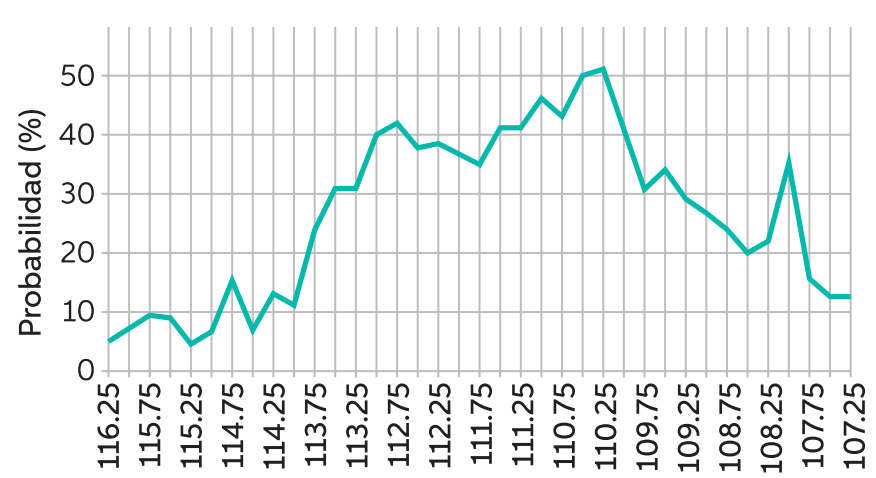

Nota: Probabilidad de que cada banda de elevación de $25 \mathrm{~cm}$ sea resubmergida por un repiquete, entre el momento de la primera exposición durante la merma anual y el momento en que el río Amazonas alcanza su nivel mínimo.

Tabla 3: Ocurrencia de repiquetes de 45 años (1968-2012) de registro diario del río Amazonas, lquitos, Perú.

\begin{tabular}{lcccc}
\hline & Nivel del río & $\begin{array}{c}\text { Probabilidad de } \\
\text { ocurrencia (\%) }\end{array}$ & $\begin{array}{c}\text { Promedio de } \\
\text { duración (días) }\end{array}$ & $\begin{array}{c}\text { Promedio de } \\
\text { magnitud (m) }\end{array}$ \\
\hline Mayo & Merma & 42.2 & 5.29 & 0.24 \\
Junio & Merma & 66.7 & 5.86 & 0.61 \\
Julio & Merma & 75.6 & 4.63 & 0.54 \\
Agosto & Merma & 95.6 & 4.59 & 0.65 \\
Septiembre & Máxima vaciante & 100 & 7.18 & 1.02 \\
Octubre & Subida & 100 & 10.1 & 1.52 \\
Noviembre & Subida & 100 & 11.95 & 1.53 \\
\hline
\end{tabular}

Notas: Probabilidad media de que los repiquetes mayores a $1 \mathrm{~cm}$ ocurran entre mayo y noviembre, como la fracción de los meses ocurridos (por ejemplo, meses de junio a lo largo de 45 años) en los que se observó al menos uno de dichos repiquetes. 
alcanzando un máximo de $>40 \%$ entre 113 y 110 $\mathrm{m}$. Dadas las típicas pendientes transversales de 1 a 3\% (hacia el centro del río) sobre los barreales en las comunidades de estudio, un aumento de medio metro del repiquete puede ahogar y matar una franja de 20 a $50 \mathrm{~m}$ de arroz recién sembrado.

\section{PÉRDIDA DE LA COSECHA}

Mientras que cualquier repiquete es potencialmente destructivo durante la temporada de cultivo de arroz en los barreales, los agricultores consideran que las reversiones del episodio de crecida del río en junio son particularmente perjudiciales. A pesar de que la probabilidad para un repiquete en junio es alta, la mayoría de los productores de arroz optan por sembrar a mediados de ese mes, independientemente del riesgo dado el alto nivel de incertidumbre y la gravedad desconocida del repiquete. La pérdida de cultivos es muy común entre los agricultores de arroz en nuestras comunidades de estudio. Durante cada una de las dos temporadas de cultivos, 2012 y 2013, el 63 y el 68\% de las familias, respectivamente, experimentaron algún déficit de cultivos en relación con su cosecha esperada (Tabla 4). En el 2013, más de la mitad de los productores de arroz experimentaron una pérdida del $50 \%$ o más de la cosecha esperada. Para cualquier agricultor de las cuatro comunidades, la probabilidad de pérdida total de cultivos era apenas inferior al $3 \%$. Los principales peligros reportados incluyen inundaciones, repiquetes, plagas, enfermedades y mala calidad del suelo. Con lo cual los agricultores experimentan pérdidas por esas múltiples causas (Tabla 2). Los repiquetes y el suelo pobre representaron el 31 y el $43 \%$ de la pérdida total de cultivos, respectivamente. Los repiquetes redujeron la producción potencial de las familias en un $50 \%$

Tabla 4: Probabilidad y severidad del déficit de arroz por peligro de sembrío en los barreales, en las comunidades de estudio del río Amazonas (2012 y 2013).

\begin{tabular}{lcccc}
\hline & \multicolumn{2}{c}{ Temporada 2012} & \multicolumn{2}{c}{ Temporada 2013 } \\
\hline & $\begin{array}{c}\text { Probabilidad de } \\
\text { déficit de cultivos } \\
(\%)\end{array}$ & $\begin{array}{c}\text { Severidad } \\
\text { promedio del } \\
\text { déficit (\%) }\end{array}$ & $\begin{array}{c}\text { Probabilidad de } \\
\text { déficit de cultivos } \\
(\%)\end{array}$ & $\begin{array}{c}\text { Severidad } \\
\text { promedio del } \\
\text { déficit (\%) }\end{array}$ \\
\hline Repiquetes & 15 & 50 & 14 & 30 \\
Suelos pobres & 11 & 62 & 14 & 33 \\
Inundación & 4 & 27 & 22 & 70 \\
Plagas/enfermedades & 11 & 40 & 11 & 27 \\
Barrancos & 4 & 50 & 0 & 0 \\
Respuesta ambigua & 19 & 24 & 8 & 37 \\
\hline Cualquier peligro de pérdida & 63 & 39 & 68 & 41 \\
\hline Número de agricultores que & 27 & & 37 & \\
siembran arroz & & & & 0 \\
\hline
\end{tabular}

Notas: El déficit se refiere a la diferencia entre la cosecha esperada y la real, según lo informado por los agricultores. El porcentaje se refiere a la proporción de agricultores que experimentan una pérdida. La severidad es el porcentaje medio de pérdida de un peligro determinado por familia, e indica la magnitud de la pérdida. La respuesta ambigua significa que el agricultor no puede atribuir la pérdida a un peligro específico, o una pérdida relacionada con otras causas, como la escasez de mano de obra para la cosecha. 
en promedio, y el suelo pobre redujo la producción en un $62 \%$. La inundación anual no representó un riesgo significativo en 2012. Durante 2011-2013, los suelos pobres - por lo general con presencia de arena- representaron la mayor parte de la pérdida de producción esperada de cultivos en las comunidades, contribuyendo con el 31\% del déficit.

Además de ahogar los cultivos, los repiquetes reducen la duración de la temporada de crecimiento del cultivo en la llanura inundable. La duración de la temporada de crecimiento varía considerablemente según la elevación de las aguas del río, pero también de un año a otro (Figura 3A). El promedio de la temporada de crecimiento a lo largo de los 45 años (1968-2012) se redujo de 218 días a $115 \mathrm{~m}$ (máximo: 342 días; mínimo: 91 días) a sólo 22 días a $109 \mathrm{~m}$ (máximo: 92 días; mínimo: 0 días). Un agricultor que cultiva su barreal a $114 \mathrm{~m}$, por ejemplo, puede esperar una temporada de crecimiento que varía entre 281 y 42 días, con una temporada media de 164 días. Cuando ocurre el repiquete los agricultores pierden no solo una porción del arroz sembrado, sino también el tiempo entre la primera siembra y la merma del agua por debajo del nivel en el que ocurrió la reversión, generalmente entre varios días y tres semanas. Este tiempo perdido no se puede recuperar, y por lo tanto la temporada de crecimiento disponible se acorta de manera efectiva. Y cuando la creciente sube y baja rápidamente, después de septiembre, la
Figura 3. Periodo de crecimiento de cultivos a lo largo del río Amazonas cerca de lquitos.

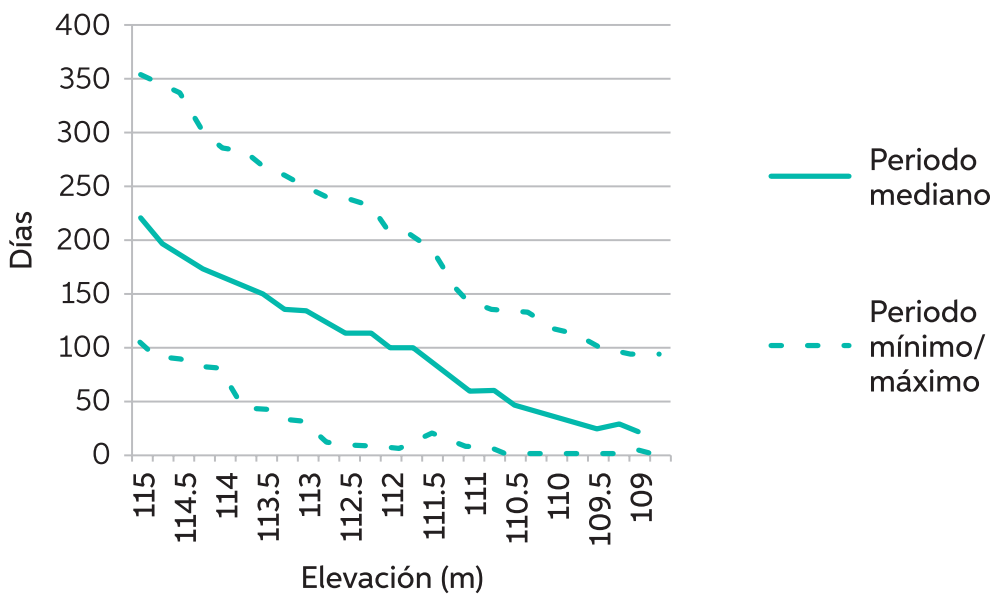

(A) Duración del periodo de crecimiento (días) por elevación del terreno

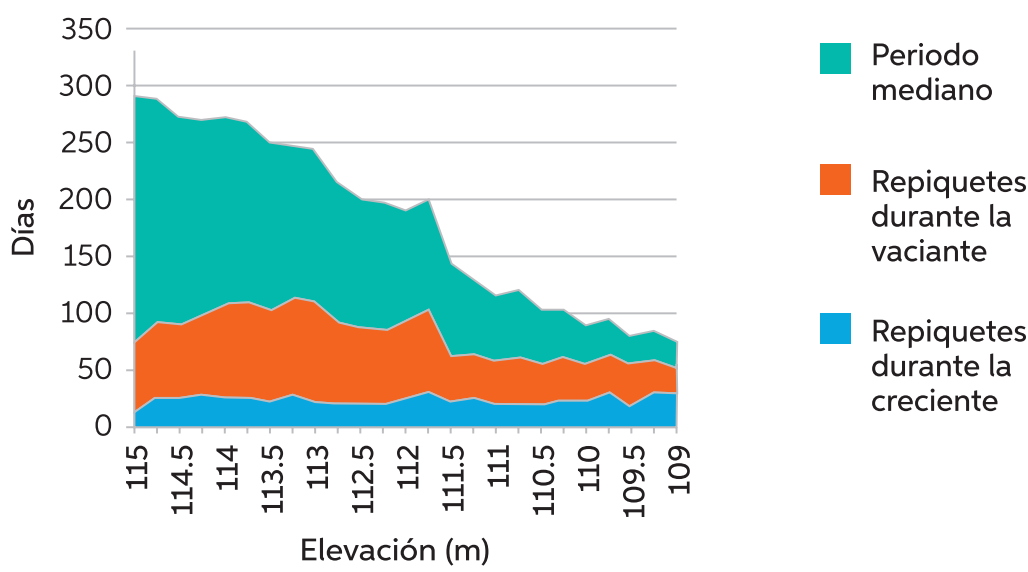

(B) Duración potencial del periodo de crecimiento por elevación del terreno

Nota: Duración real de la temporada de crecimiento agregada a los días perdidos por repiquetes para indicar la duración potencial del periodo.

duración de la temporada de crecimiento también se acorta. La Figura 3B ilustra la importancia de los repiquetes a medida que baja la creciente y luego aumenta en relación con la duración de la temporada de crecimiento, y entre sí.

Para cultivos de la llanura inundable como el arroz, que requiere de 75 a 165 días para madurar según la variedad, la ventana de oportunidad para la maduración entre junio y octubre se reduce considerablemente debido a los repiquetes. La 
Figura 4. Probabilidad que el periodo de crecimiento de cultivos llegue a 75-150 días por elevación del terreno.

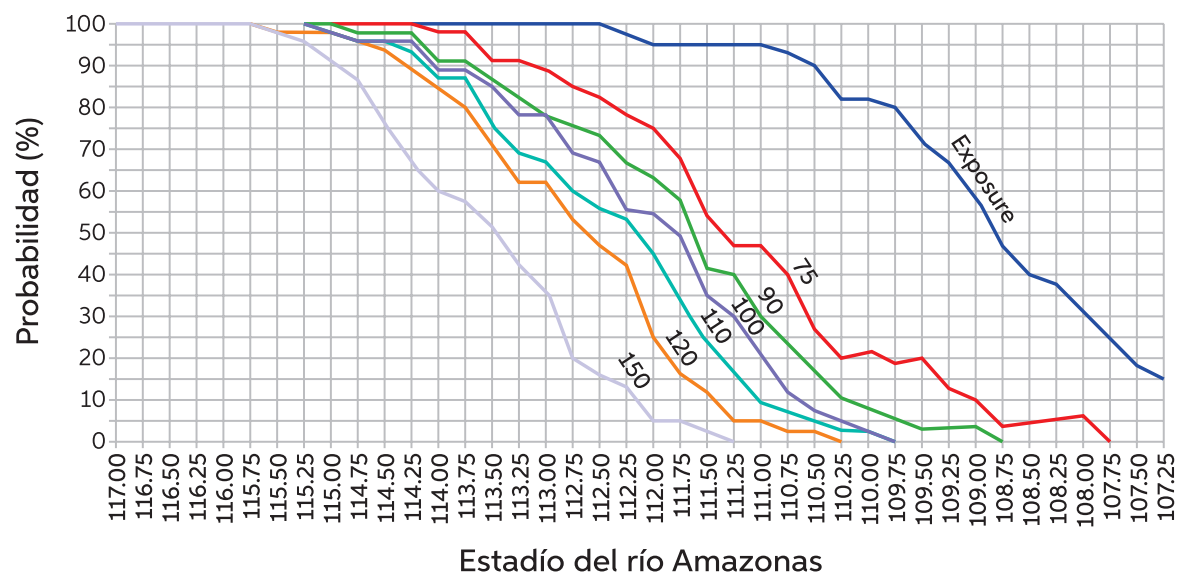

ascendió a 115\% del ingreso total en ese año, en promedio. En otras palabras, si se hubiera realizado la producción total de arroz, el ingreso promedio de las familias se habría duplicado. El alto riesgo y volatilidad en la cosecha concuerda con los altos rendimientos potenciales. En años en que las familias cosechan su cultivo completo con rendimientos óptimos, el ingreso del arroz claramente representaría una verdadera

Figura 4 muestra la probabilidad de exposición de la tierra inundada a elevaciones entre $107.25 \mathrm{~m}$ y $117.00 \mathrm{~m}$ y la probabilidad que la temporada de crecimiento alcance a los cultivos que requieren de 75 a 150 días para su madurez. A una elevación de $111.50 \mathrm{~m}$, la probabilidad de exposición al barreales es de alrededor del 95\%, pero la probabilidad de lograr una temporada de crecimiento de 75 días sería solo del 55\% y menos del $5 \%$ para una temporada de crecimiento de 150 días. No es sorprendente entonces que los agricultores prefieran variedades de arroz de maduración más rápida, como Dos meses y medio, Aguja, Tres mesinos y Milagrito (75-90 días) en lugar de variedades de mayor periodo de maduración pero de mayor rendimiento promovidas por el Estado, incluida Capirona, Esperanza o Inti (120-165 días).

Las pérdidas económicas asociadas con la caída de la cosecha son sustanciales para las familias que, generalmente, son pobres. En 2013, la diferencia entre lo esperado y lo real fue de $93,440 \mathrm{~kg}$ en las cuatro comunidades se valuó en aproximadamente US\$ 83,000 (List \& Coomes, 2017). El déficit medio fue de $3,738 \mathrm{~kg}$ equivalente a US\$3,338 en pérdidas promedio al ingreso por familia. Para las familias que sufrieron pérdidas de arroz en 2013, el valor del cultivo bonanza, mientras que las pérdidas por peligros naturales pueden traer considerables dificultades económicas a las familias agricultoras.

\section{OPCIONES DE REDUCCIÓN DEL RIESGO DE INUNDACIÓN}

Las altas pérdidas potenciales apuntan a la necesidad de aplicar instrumentos para reducir el riesgo de las inundaciones entre los agricultores de las llanuras inundables. Se aplicaron cuatro instrumentos en un experimento con agricultores de las cuatro comunidades involucradas en el proyecto, con el propósito de evaluar i) el método de estimación hipotético (WTP) para el pago del crédito; ii) las variedades de arroz tolerantes a las inundaciones; iii) los servicios de información fluvial; $y$, iv) el seguro contra inundaciones. Los resultados muestran que el $40 \%$ de los encuestados considera que el crédito sería útil en tiempos de inundación (Figura 5). El endeudamiento fue reportado como el principal elemento negativo persuasivo. Dos tercios de los agricultores creen que las variedades de arroz tolerantes a las inundaciones, como el arroz Scuba de Asia (ver Dar et al., 2013), serían útiles frente a las inundaciones. Los agricultores estarían dispuestos a pagar US\$ $1.60 / \mathrm{kg}$ de semilla, equivalente al doble del costo 
Figura 5. Disposición de pagar (WTP) por instrumentos de adaptación a las inundaciones.

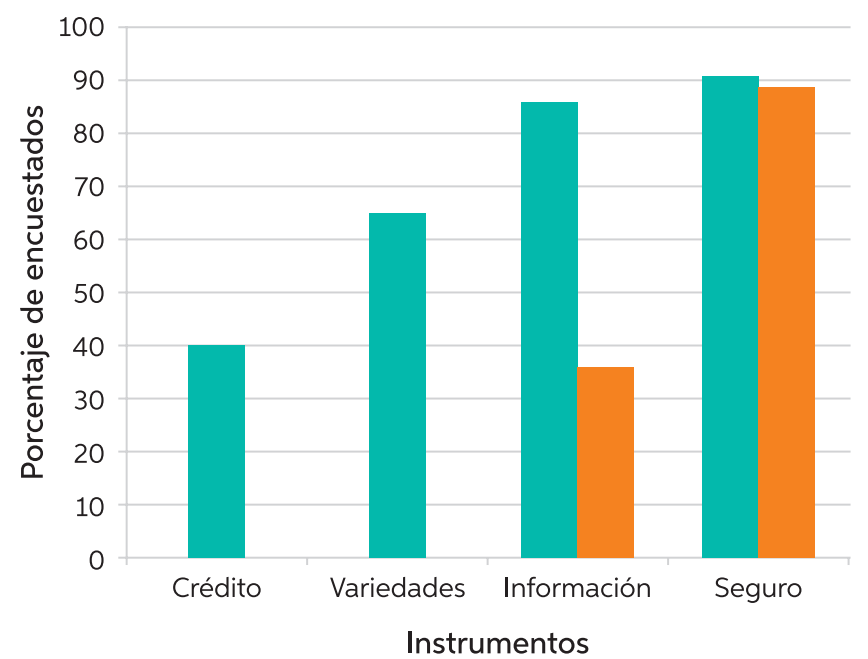

Disposición a pagar

$\begin{array}{llll}\text { Promedio } & \$ 1.60 / \mathrm{kg} & \$ 8.27 / \text { año } & \$ 7.21 / \mathrm{mes} / \mathrm{ha} \\ \text { Mínimo } & \$ 0.18 / \mathrm{kg} & \$ 0.71 / \text { año } & \$ 0.36 / \mathrm{mes} / \mathrm{ha} \\ \text { Máximo } & \$ 7.14 / \mathrm{kg} & \$ 35.71 / \text { año } & \$ 35.71 / \mathrm{mes} / \mathrm{ha} \\ \mathrm{N} & 50 & 25 & 67\end{array}$

Notas: WTP=Willingness to pay. El tipo de cambio era de 2.8 NS/\$US, julio 2014

\section{DISCUSIÓN}

Este estudio realizado cerca de Iquitos examina una fuente importante de riesgo de cultivos: las reversiones inesperadas 0 repiquetes. Durante la época de vaciante, los agricultores enfrentan un promedio de 5 a 6 repiquetes por año entre los meses de primera siembra de arroz (junio y julio), cada uno con una duración promedio de cinco días y con una elevación promedio de $0.5 \mathrm{~m}$. Los repiquetes no solo pueden ahogar los cultivos recién plantados en el canal activo del río, sino que también acortan significativamente la duración efectiva de la temporada de crecimiento. En los terrenos elevados sembrados de arroz

promedio de la semilla de arroz en la región (US\$ $0.79 / \mathrm{kg}$ ). El $86 \%$ de la muestra consideró que los servicios de información fluvial eran útiles; sin embargo, solo el $36 \%$ estaría dispuesto a contribuir financieramente a dicho servicio. Aquellos agricultores que deseen pagar por la información aportarían anualmente en promedio US\$ 8.00, apenas el $0.4 \%$ de su ingreso familiar promedio anual. En el caso del seguro contra inundaciones agrícolas, el $95 \%$ de las familias encuestadas nunca había oído hablar de él antes de la entrevista. Después de la breve explicación, el 91\% de los encuestados indica que el seguro sería útil para estar al tanto de las inundaciones, y el $89 \%$ estaría dispuesto a pagar un plan de seguro. En promedio, los encuestados estarían dispuestos a pagar como máximo US\$ $7.21 /$ mes /ha o US\$ 87.00/año /ha (mediana de US\$ 43/ año/ha).
(116-112 msnm) cada año se pierden en promedio 80 días potenciales por repiquetes, 20 durante la temporada de vaciante y 60 durante la subida. Esta pérdida es sustancial, ya que las variedades de arroz locales requieren una temporada de crecimiento de 75-165 días. Curiosamente, las prácticas agrícolas locales parecen reflejar un análisis de riesgo implícito basado en la experiencia agrícola. Mientras que la probabilidad de una nueva sumersión por un repiquete es generalmente inferior al $10 \%$ en elevaciones de canal por encima de $114 \mathrm{~m}$ el riesgo de inundaciones por repiquetes aumenta rápidamente al 50\% con una caída de sumersión de $1 \mathrm{~m}$ por debajo de $111.75 \mathrm{~m}$. Los agricultores actualmente dejan de sembrar arroz a $112 \mathrm{~m}$, donde la probabilidad condicional de tener al menos una temporada de cultivo ininterrumpida 
de 100 días cae por debajo del 50\%, si la siembra se realiza después del 1 de agosto.

Los agricultores cerca de Iquitos claramente enfrentan un alto riesgo de pérdida de cultivos de arroz en terrenos con sedimentos de limo en el canal activo. Nuestros hallazgos sugieren que los repiquetes y las condiciones edáficas pueden ser tan importantes o más que el riesgo de inundación anual; hecho que se destaca más comúnmente en la investigación sobre la agricultura de recesión de inundaciones en la Amazonia (Hiraoka, 1985; Chibnik, 1994; Labarta et al., 2007). En efecto, los agricultores de arroz que trabajan la tierra en el canal activo del río enfrentan múltiples peligros, que incluyen inundaciones tempranas, plagas y enfermedades, desbarrancamientos y las malas condiciones del suelo. La escasez de la cosecha refleja la considerable heterogeneidad en las condiciones edáficas en este entorno fluviogeomorfológico dinámico, a pesar de la mayor fertilidad del suelo en relación con las tierras de la altura.

Entre los instrumentos de mitigación de riesgos contemplados, los agricultores locales consideraron que las variedades tolerantes a las inundaciones se encuentran entre las menos interesantes. La inundación compromete los cultivos en pie de múltiples maneras, más allá de detener la maduración por ahogamiento, como por la depredación de semillas y consumo de cultivos por peces y el daño de los cultivos por la corriente del río. Si bien las variedades de arroz tolerantes a las inundaciones pueden soportar la inmersión en agua, es posible que no sobrevivan a la capa de sedimentos y a los riesgos relacionados con las inundaciones que presenta el río Amazonas. La mayoría de los agricultores de la muestra también preferían las variedades locales con tiempos de maduración cortos entre 75 y 90 días, debido al riesgo de inundación.

Los servicios de información son de interés para muchos agricultores, pero sólo alrededor de un tercio de los encuestados estaría dispuesto a pagar por dicho servicio. Durante las entrevistas, el personal del servicio meteorológico peruano (Servicio Nacional de Meteorología e Hidrología del Perú - SENAMHI) manifestó su interés en desarrollar un sistema más eficaz de alerta temprana de inundaciones, pero destacó que la tecnología de la comunicación presentaba una limitación crítica. En la muestra del estudio, poco más de la mitad de las familias tenía una radio y sólo una cuarta parte tenía un teléfono celular en un área donde la recepción celular se da en el mejor de los casos con interrupciones. Aunque es poco probable que un sistema de alerta temprana pueda predecir la magnitud o la duración de un repiquete con suficiente antelación, dada la ubicación espacialmente desigual delas estaciones de mediciones meteorológicas e hidrográficas y la naturaleza abrupta de los eventos de reversión de inundaciones (Coomes et al., 2016); un sistema de advertencia, no obstante, sería valioso para ayudar a los agricultores de la llanura inundable en la etapa inicial crucial de la siembra, cerca de los cultivos de maduración. Además, tendría información relevante para comunicar a los agricultores, al comienzo de la creciente, sobre la ocurrencia y la influencia de la alta temperatura en la superficie del mar en el Atlántico y el Pacífico, que influyen en las condiciones de inundación y sequía en la Amazonía.

Los agricultores mostraron una fuerte preferencia por un seguro de inundación basado en índices: el 91\% consideró que sería útil y el 89\% estaría dispuesto a pagar por ello. Los agricultores estarían dispuestos a pagar un promedio de US\$ 87/año/ha para asegurar los cultivos de la pérdida por inundación, un gasto significativo para los productores de arroz en la región. La producción de arroz en los barreales y la forma de relieve más baja cultivada hizo que las familias en nuestro estudio obtuvieran un promedio de US\$ 2,315/ año/ha, sin considerar el costo de los insumos. 
Como tal, los agricultores están dispuestos a pagar cerca del 4\% de las ganancias de la producción de arroz en un plan de seguro, equivalente al 2.5\% del ingreso promedio anual de las familias. Este resultado debe considerarse con la advertencia de que a los encuestados se les preguntó acerca de un producto hipotético y, como tal, WTP puede ser exagerada. Sin embargo, los resultados son sugerentes. Las entrevistas con un proveedor de seguros en Lima, afiliada con el suministro de otros productos de seguros indexados en Perú, expresaron un gran interés en desarrollar un producto de seguros basado en índices para la llanura inundable del río Amazonas. El interés demostrado por los agricultores, la experiencia comercial peruana con seguros climáticos y las condiciones medibles de riesgo ambiental (con registro de más de 45 años de los niveles diarios de agua del río) que atañen a la pérdida de cultivos, sugieren que un producto de seguro basado en un índice del reporte de inundaciones podría ser útil para los agricultores que trabajan en las llanuras inundables y canal activo del río Amazonas (List et. al., 2019).

Se necesita promover más investigación, para evaluar las posibles medidas de mitigación de riesgos que pueden ser más útiles y económicamente viables para las familias agricultoras arroceras, particularmente frente al cambio climático, lo cual se prevé que incremente la duración del proceso de la temporada de inundaciones y la extensión de las inundaciones a lo largo de los ríos en la Amazonía occidental; y para identificar las barreras políticas locales, regionales y estatales, para su adopción a la mitigación de tales riesgos. Los agricultores entrevistados en nuestro estudio expresaron su interés en encontrar nuevas formas de mitigar el riesgo del peligro natural. El seguro basado en índices del clima sería una de las herramientas de adaptación estratégica más amplia y de múltiples aplicaciones (ver Carter et al., 2014; Surminski et al., 2016), para reducir la incertidumbre de las inundaciones que padecen los agricultores de las llanuras inundables y para desbloquear el potencial completo de desarrollo agrícola de las tierras inundables a lo largo de la cuenca Amazónica.

\section{AGRADECIMIENTOS}

Los autores desean agradecer al Cap. Carlos Holguín Valdivia del Servicio de Hidrografía y Navegación de la Amazonía (SEHINAV) en Iquitos por facilitar los datos diarios del nivel de agua del río. Agradecemos, además, a las personas de las comunidades de estudio, por su participación en la investigación y a nuestros asistentes de campo, Mayra Quevedo Guerrero y Carlos Rengifo Upiachihua. Michael Templeton y Adrian Lepoutre proporcionaron asistencia en la compilación y análisis de datos. El consejo y la orientación de Michel Lapointe también fueron muy apreciados. El texto fue traducido hábilmente por Santiago Rivas Panduro a quien también le expresamos nuestro agradecimiento.

\section{BIBLIOGAFÍA CITADA}

Carter, M.; de Janvry, A.; Sadoulet, E.; Sarris, A. 2014. Index-based weather insurance for developing countries: A review of evidence and a set of propositions for up-scaling. Working Paper Development Policies. Background paper. Fondation pour les Etudes et Recherches sur le Développement International, Paris. 33 pp.

Chibnik, M. 1994. Risky rivers: the economics and politics offloodplain farming in the Amazon. The University of Arizona Press, Tucson. 267 pp.

Coomes, 0.T.; Lapointe, M.; Templeton, M; List, G. 2016. Amazon river flow regime and flood recessional agriculture: flood stage reversals 
and risk of annual crop loss. Journal of Hydrology, 539: 214-222.

Dar, M.H.; De Janvry, A.; Emerick, K.; Raitzer, D.; Sadoulet, E. 2013. Flood-tolerant rice reduces yield variability and raises expected yield, differentially benefitting socially disadvantaged groups. Scientific Reports, 3 (3315): 1-8.

Denevan, W.M. 1984. Ecological heterogeneity and horizontal zonation of agricultural in the Amazon floodplain. In: Schmink, M.; Woods, C. (Eds). Frontier Expansion in Amazonia. University of Florida Press, Gainesville, p. 311336.

Humala Tasso, OM; Von Hess La Serna, M; Rheineck Piccardo, J.L.; Muro Ventura, J.A.; Salazar Córdova, R. 2013. El arroz. Principales aspectos de la cadena agroproductiva. Dirección General de Competitividad Agraria, Ministerio de Agricultura, Lima. 36 pp.

Labarta, R.A.; White, D.; Leguía, E.; Guzmán, W.; Soto, J. 2007. La agricultura en la Amazonia ribereño del rio Ucayali. ¿Una zona productiva pero poco rentable? Acta Amazonica, 37 (2): 177-186.

Langerwisch, F.; Rost, S.; Gerten, D.; Poulter, B.; Cramer, W. 2013. Potential effects of climate change on inundation patterns in the Amazon basin. Hydrology and Earth System Sciences, 17: 2247-2262.

List, G. 2016. Agriculture and the risk of crop loss in the Amazon river floodplain of Peru. Masters Thesis, McGill University, Montreal. 139 pp.

List, G.; Coomes, O.T. 2017. Natural hazards and risk in rice cultivation along the upper Amazon river. Natural Hazards, 87 (1): 165-184.

List, G.; Laszlo, S.; Coomes, O.T. (2019). Mitigating risk for floodplain agriculture in Amazonia: a role for index-based flood insurance. Climate and Development (in press).

Mendoza, A.; Abad, J.D.; Frias, C.E.; Ortals, C.; Paredes, J.; Montoro, H.; Vizcarra, J.; Simon, C.; Soto-Cortés. G. 2016. Planform dynamics of the Iquitos anabranching structure in the Peruvian Upper Amazon River. Earth Surface Processes and Landforms, 41: 961-970.

Petrick, C. 1978. The complementary function of floodlands for agricultural utilization. Applied Sciences and Development, 12: 26-46.

Pinedo-Vásquez, M.; Barletti Pasqualle, M.; Del Castillo Torres, D.; Coffey, K. 2002. A tradition of change: the dynamic relationship between biodiversity and society in sector Muyuy, Peru. Environment and Science Policy, 5 (1): 43-53.

Ríos Arévalo, M. 2005. Agrobiodiversification of sand bars and mudflats, and their role in the riverine rural economy of the Peruvian Amazon. Masters Thesis, Nucleo de Altos Estudos Amazônicos, Universidade Federal do Pará, Belem. 161 pp.

Ronchail, J.; Espinoza, J.C.; Drapeau, G.; Sabot, M.; Cochonneau, G.; Schor, T. 2018. The flood recession period in Western Amazonia and its variability during the 1985-2015 period. Journal of Hydrology: Regional Studies, 15: 1630.

Smith, N.J.H.; Serrão, E.A.S.; Alvim, P.T.; Falesi, I.C. 1995. Amazonia - resiliency and dynamism of the land and its people. United Nations University Press, Tokyo, 252pp.

Surminski, S.; Bouwer, L. M.; Linnerooth-Bayer, J. 2016. How insurance can support climate resilience. Nature Climate Change, 6 (4), 333334.

Recibido: 24 de marzo de 2019 Aceptado para publicación: 20 de setiembre de 2019 